International Journal of Pure and Applied Mathematics

Volume 89 No. 4 2013, 553-558

ISSN: 1311-8080 (printed version); ISSN: 1314-3395 (on-line version)

url: http://www.ijpam.eu

doi: http://dx.doi.org/10.12732/ijpam.v89i4.9

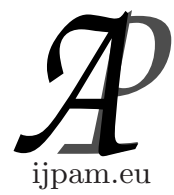

\title{
CO-IDEALS IN TERNARY SEMIGROUPS
}

\author{
T. Gaketem \\ Department of Mathematics \\ School of Science \\ Phayao University \\ Phayao, 56000, THAILAND
}

\begin{abstract}
In this page we study properties of co-ideal in the ternary semi$\operatorname{group}\left\langle\mathbb{Z}_{0}^{+}, \cdot\right\rangle$.
\end{abstract}

Key Words: ternary semigroup, ternary semiring, co-ideal, subtractive coideal

\section{Introduction}

In [1]. Cayley and Sylvester along with several other mathematicians, in the 19th century considered ternary algebraic structures and cubic relations. The nary structures, which are the generalizations of ternary structures create hopes because of their possible applications in Physics. A few important physical applications have been recorded in Ternary semigroups exhibit natural examples of ternary algebras. Also S. Kar [7] studied the ideal theory in the ternary semiring $\left\langle\mathbb{Z}_{0}^{-}, \cdot\right\rangle$.

In this paper, we introduce the concept of a co-ideal and study properties of a co-ideals of ternary semigroups $\left\langle\mathbb{Z}_{0}^{+}, \cdot\right\rangle$.

Received: August 25, 2013

(c) 2013 Academic Publications, Ltd. url: www.acadpubl.eu 


\section{Preliminaries}

Definition 2.1. [7, p.192] A non-empty set $S$ together with a ternary operation, called ternary multiplication, denoted by juxtaposition, is said to be a ternary semigroup if $(a b c) d e=a(b c d) e=a b(c d e)$ for all $a, b, c, d, e \in S$.

Definition 2.2. $\quad[7$, p.192] An element $e$ of a ternary semigroup $S$ is called

(1) a left identity (or left unital element) if eex $=x$ for all $x \in S$,

(2) a right identity (or right unital element) if $x e e=x$ for all $x \in S$,

(3) a lateral identity (or lateral unital element) if exe $=x$ for all $x \in S$,

(4) a two-sided identity (or bi-unital element) if $e e x=x e e=x$ for all $x \in S$,

(5) an identity (or unital element) if eex $=e x e=x e e=x$ for all $x \in S$.

Example 2.3. Let $\left\langle\mathbb{Z}_{0}^{+}, \cdot\right\rangle$ be the set of all non-positive integers. Then with the usual ternary multiplication. $\left\langle\mathbb{Z}_{0}^{+}, \cdot\right\rangle$ forms a ternary semigroup with zero element 0 and identity element 1

Definition 2.4. [7, p.192] A non-empty subset $T$ of a ternary semigroup $S$ is called a ternary subsemigroup if $t_{1} t_{2} t_{3} \in T$ for all $t_{1}, t_{2}, t_{3} \in T$.

Definition 2.5. [7, p.192] A ternary subsemigroup $I$ of a ternary semigroup $S$ is called

(1) a left ideal of $S$ if $S S I \subseteq I$,

(2) a right ideal of $S$ if $I S S \subseteq I$,

(3) a lateral ideal of $S$ if $S I S \subseteq I$,

(4) a two-sided ideal of $S$ if $I$ is both left and right ideal of $S$,

(5) an ideal of $S$ if $I$ is a left, a right, a lateral ideal of $S$.

An ideal $I$ of a ternary semigroup $S$ is called a proper ideal if $I \neq S$. 


\section{Co-Ideals in Ternary Semigroup}

We define co-ideal of a ternary semigroup, and investigate it properties.

Definition 3.1. A non-empty subset $I$ of a ternary semigroup $S$ is called a co-ideal if

(1) $a, b, c \in I$ implies $a b c \in I$,

(2) $a \in I, s \in S$ implies as $\in I$.

Definition 3.2. A co-ideal $I$ of a ternary semigroup $S$ is called subtractive if $a, b, a b c \in I, c \in S$, then $c \in I$

Theorem 3.3. Let $I, J$ be a co-ideal of a ternary semigroup $S$. Then

(1) $I \cap J$ is a co-ideal of $S$,

(2) $I J$ is a co-ideal of $S$,

(3) $I J \subseteq I \cap J$.

Proof. (1) Let $a, b, c \in I \cap J$ then $a b c \in I$, since $I, J$ is a co-ideal and so $a b c \in J$. Hence $a b c \in I \cap J$.

Let $a \in I \cap J$ and $r \in S$ then $a s \in I$ and $a s \in J$, since $I, J$ is a co-ideal of $S$. Thus as $\in I \cap J$. Hence $I \cap J$ is a co-ideal of $S$.

(2) Let $x=a b, y=a^{\prime} b^{\prime}, z=a^{*} b^{*} \in I J$. Then

$$
\begin{aligned}
x y z= & (a b)\left(a^{\prime} b^{\prime}\right)\left(a^{*} b^{*}\right) \\
= & {\left[\left(a a^{\prime}\right)\left(a b^{\prime}\right)\left(b a^{\prime}\right)\left(b b^{\prime}\right)\right]\left(a^{*} b^{*}\right) } \\
= & \left(\left[\left(a a^{\prime}\right)\left(a^{*} b^{*}\right)\right]\left[\left(a b^{\prime}\right)\left(a^{*} b^{*}\right)\right]\left[\left(b a^{\prime}\right)\left(a^{*} b^{*}\right)\right]\left[\left(b b^{\prime}\right)\left(a^{*} b^{*}\right)\right]\right) \\
= & {\left[\left(a a^{*}\right)\left(a b^{*}\right)\left(a^{\prime} a^{*}\right)\left(a^{\prime} b^{*}\right)\right]\left[\left(a a^{*}\right)\left(a b^{*}\right)\left(b^{\prime} a^{*}\right)\left(b^{\prime} b^{*}\right)\right] } \\
& {\left[\left(b a^{*}\right)\left(b b^{*}\right)\left(a^{\prime} a^{*}\right)\left(a^{\prime} b^{*}\right)\right]\left[\left(b a^{*}\right)\left(b b^{*}\right)\left(b^{\prime} a^{*}\right)\left(b^{\prime} b^{*}\right)\right] } \\
= & {\left[\left(a a^{*}\right)\left(a b^{*}\right)\left(a^{\prime} a^{*}\right)\left(a^{\prime} b^{*}\right)\left(a a^{*}\right)\left(a b^{*}\right)\left(b^{\prime} a^{*}\right)\left(b^{\prime} b^{*}\right)\right.} \\
& \left.\left(b a^{*}\right)\left(b b^{*}\right)\left(a^{\prime} a^{*}\right)\left(a^{\prime} b^{*}\right)\left(b a^{*}\right)\left(b b^{*}\right)\left(b^{\prime} a^{*}\right)\left(b^{\prime} b^{*}\right)\right] \\
= & \left(a a^{*}\right)^{2}\left(a b^{*}\right)^{2} a^{\prime}\left(a^{*} b^{*}\right) b^{\prime}\left(a^{*} b^{*}\right) b\left(a^{*} b^{*}\right) a^{\prime}\left(a^{*} b^{*}\right) b\left(a^{*} b^{*}\right) b^{\prime}\left(a^{*} b^{*}\right) \\
= & \left(a a^{*}\right)^{2}\left(a b^{*}\right)^{2} a^{\prime} b^{\prime} b a^{\prime} b b^{\prime}\left(a^{*} b^{*}\right) \\
= & \left(a a^{*}\right)^{2}\left(a b^{*}\right)^{2} a^{\prime 2} b^{2} b^{\prime 2}\left(a^{*} b^{*}\right) .
\end{aligned}
$$

Since $\left(a^{*} b^{*}\right) \in I J$ we have $x y z \in I J$.

Let $r \in S$ then $x r=(a b) r \in I$, since $I$ is a co-ideal of $S$. Hence $I J$ is a co-ideal of $S$. 
(3) Let $x=a b \in I J$ where $a \in I, b \in J$. Now $a \in I, b \in S$ and $I$ is a co-ideal implies $x=a b \in I$. Similarly $x \in J$. Hence $x \in I \cap J$ so $I J \subseteq I \cap J$.

Corollary 3.4. Every ideal is a co-ideal.

Proof. Let $I$ is an ideal of ternary semigroup of $S$ and $a, b, c \in I$. Then $a b c \in I$. Let $a \in I$ and $s \in S$ then $a s \in I$, since $I$ is an ideal of $S$. Thus $I$ is a co-ideal of $S$.

Theorem 3.5. Let $I, J$ be a subtractive co-ideal of a ternary semigroup $S$. Then $I \cap J$ is a subtractive co-ideal of $S$.

Proof. By Theorem 3.3(1) we have $I \cap J$ is a co-ideal of $S$. If $a, b, a b c \in I \cap J$, $c \in S$ then $c \in I \cap J$, since $I, J$ is a subtractive co-ideal of $S$.

Lemma 3.6. Let $I, J$ be a subtractive co-ideal of a ternary semigroup $S$ and $a \in I, b \in J, c \in I \cap J$. Then the following conditions are equivalent

(1) $a b c \in I \cap J$,

(2) $a \in I \cap J$ or $b \in I \cap J$,

(3) $a, b \in I$ or $a, b \in J$.

Proof. $(1) \Rightarrow(2)$ Let $a b c \in I \cap J$. Without loss of generality assume that $a b c \in I$. Since $I$ is a subtractive co-ideal we have $b \in I$. Hence $b \in I \cap J$.

$(2) \Rightarrow(3)$ Let $a \in I \cap J$ or $b \in I \cap J$. We must to show that $a, b \in I$ or $a, b \in J$

Case 1. If $a \in I \cap J$ then $a \in I$ and $a \in J$

Case 2. If $b \in I \cap J$. then $b \in I$ and $b \in J$

From case1.,2. we have $a, b \in I$ or $a, b \in J$.

$(3) \Rightarrow(1)$ Let $a, b \in I$ or $a, b \in J$ then $a b \in I$ or $a b \in J$. Since $I, J$ is a subtractive co-ideal and $c \in I \cap J$ we have $a b c \in I \cap J$.

Theorem 3.7. Let $I, J$ be subtractive co-ideals of a ternary semigroup $S$ and $A$ be ideal of $S$ such that $A \cap I \cap J \neq$. Then $A \subseteq I \cap J$ if and only if $A \subseteq I$ or $A \subseteq J$

Proof. Let $c \in A \cap i \cap J$. Let $A \subseteq I \cap J$ and $A \nsubseteq I$. Choose $a \in A$ such that $a \notin I$. Then $a \in J$. We claim that $A \cap I \subseteq J$. Let $b \in A \cap I$. Now $a, b, c \in A$ and $A$ is a co-ideal implies that $a b c \in A \subseteq I \cap J$. By Lemma 3.6 $a, b, \in I$ or $a, b \in J$. Then $a, b \in J$, since $a \notin I$. Hence $A \cap I \subseteq J$. Now $A=A \cap(I \cap J)=(A \cap I) \cap(A \cap J) \subseteq J \cap J=J$. Converse is trivial. 
Corollary 3.8. Let $I, J$ be subtractive co-ideals of a ternary semigroup $S$ such that $I \cap J \neq$. Then $I \cap J$ is co-ideal of $S$ if and only if $I \subseteq J$ or $J \subseteq I$. if and only if $A \subseteq I$ or $A \subseteq J$

Proof. Let $I \cap J$ is a co-ideal of $S$. Now $I \cap J \subseteq I \cap J$ and hence by Theorem 3.7, $I \cap J \subseteq J$ or $I \cap J \subseteq J$. Then $A \subseteq I$ or $A \subseteq J$.

Conversely let $A \subseteq I$ or $A \subseteq J$ to show that $I \cap J$ is a co-ideal. Let $a, b, c \in I \cap J$ then $a b c \in I \cap J$, since $I, J$ is a subtractive co-ideals. Let $a \in I \cap J$ and $s \in S$ by Theorem 3.5, $I \cap J$ is a subtractive. Thus ar $\in I \cap J$. Hence $I \cap J$ is a co-ideal.

\section{Co-Ideals in Ternary Semigroup $\left\langle\mathbb{Z}_{0}^{+}, \cdot\right\rangle$}

We study properties of co-ideal in ternary semigroup $\mathbb{Z}_{0}^{+}$

Lemma 4.1. $I_{n}=\left\{a \in \mathbb{Z}_{0}^{+}: a \leq n\right\}$ is a co-ideal.

Proof. Let $a, b, c \in I_{n}$ then $a, b, c \in \mathbb{Z}_{0}^{+}$. Thus $a b c \in \mathbb{Z}_{0}^{+}$so $a b c \in I_{n}$. $\mathbb{Z}_{0}^{+}$

Let $a \in I_{n}$ and $r \in S$ then $a r \in \mathbb{Z}_{0}^{+}$. Thus ar $\in I_{n}$. Hence $I_{n}$ is a co-ideal of

Theorem 4.2. A non-empty subset $I$ of the ternary semigroup $\left\langle\mathbb{Z}_{0}^{+}, \cdot\right\rangle$ is a co-ideal if and only if $I=I_{n}$

Proof. $(\Rightarrow)$ Assume that $I$ is a co-ideal of $\left\langle\mathbb{Z}_{0}^{+}, \cdot\right\rangle$. We must show that $I=I_{n}$ Since $I$ is a non-empty, $I$ has the largest element say $n$. Let $x \in I_{n}$ then $x \leq n$ and $x=n y$ for some $y \in \mathbb{Z}^{+}$. Now $x \in I$, since $I$ is a co-ideal. Hence $I_{n} \subseteq I$. But $I \subseteq I_{n}$. So $I=I_{n}$.

$(\Leftarrow)$ Assume that $I=I_{n}$ by Lemma4.1 then $I_{n}$ is a co-ideal of $\mathbb{Z}_{0}^{+}$. Thus $I$ is a co-ideal of $\left\langle\mathbb{Z}_{0}^{+}, \cdot\right\rangle$.

\section{Acknowledgments}

The author is very grateful to the anonymous referee for stimulating comments and improving presentation of the paper. 


\section{References}

[1] S. Bashir, M. Shabir, Pure ideals in ternary semigroups, Quasigroups and Related Systems, 17 (2009), 149-160.

[2] J.N. Chaudhari, K.J. Ingale, Prime avoidance theorem for co-ideals in semirings, Research Journal of Pure Algebra, 1, No. 9 (2011), 213-216.

[3] J.N. Chaudhari, H.P. Bendale, Co-ideals in ternary semirings, Research Journal of Pure Algebra, 2, No. 9 (2012), 62-65.

[4] E. Hewitt, H.S. Zuckerman, Ternary operations and semigroups, Proc. Sympos. Wayne State Univ., Detroit (1968), 55-83.

[5] R. Kerner, Ternary algebraic structures and their applications in Physics, Univ. PM. Curie Preprint, Paris (2000), Arxiv math-Ph/0011023.

[6] S. Kar, Ideal theory in the ternary semiring $\left\langle\mathbb{Z}_{0}^{-}, \cdot\right\rangle$, Bull. Malaysian. Math. Sci. Soc., To Appear.

[7] S. Kar, B.K. Maity, Congrences on ternary semigroups, Joural of the Chungcheong Mathenatical Sociery, 20 (2007), 191-201.

[8] M. Shabir, M. Bano, Prime bi-ideals in ternary semigroups, Quasigroups and Related Systems, 16 (2008), 239-256. 